\title{
Abordagem multidisciplinar ao paciente oncológico adulto e idoso ostomizado: uma revisão narrativa
}

\author{
Multidisciplinary approach for the adult and elderly oncological ostomized patient: a \\ narrative review
}

Enfoque multidisciplinario para pacientes oncológicos adultos y ancianos ostomizados: una revisión narrativa

Silvana Bastos Cogo ${ }^{1}$, Márcio Rossato Badke ${ }^{1}$, Graciela Dutra Sehnem ${ }^{1}$, Ariele Priebe Reisdorfer ${ }^{1}$, Raquel Karlinski Almeida ${ }^{1}$, Stéfani Fernanda Schumacher ${ }^{1}$, Ariélen Ferigollo ${ }^{1 *}$, Andrei Pompeu Antunes ${ }^{1}$, Aline Gomes Ilha ${ }^{1}$, Luiza Carolina Santos Malheiros ${ }^{1}$.

\section{RESUMO}

Objetivo: Realizar uma revisão sobre os aspectos biopsicossociais bem como a atuação da esquipe multidisciplinar frente aos pacientes adultos e idosos oncológicos em uso de ostomias. Revisão bibliográfica: Inicialmente, foram selecionadas 30 publicações que apresentaram os critérios de inclusão. Posteriormente, a análise dos dados disponíveis na literatura nacional e internacional possibilitaram a descrição das atuais perspectivas epidemiológicas que evidenciam o crescimento dos novos casos de câncer associados a mudanças no perfil sociodemográfico da população. E assim, de forma concomitante, o crescimento das sequelas ocasionadas pelo tratamento antineoplásico, com destaque para o processo de ostomização. Esta revisão possibilitou a descrição dos tipos de ostomias, quais as possíveis complicações que o paciente pode apresentar e como se dá o processo de adaptação a esta nova condição. Considerações finais: $O$ estudo demonstra a importância da abordagem multiprofissional na garantia da qualidade de vida na perspectiva biopsicossocial do paciente adulto e idoso oncológico ostomizado.

Palavras-chave: Estomia, Neoplasias, Equipe de assistência ao paciente.

\begin{abstract}
Objective: A review was realized about the biopsychosocial aspects as well as the performance of the multidisciplinary team by the relation with the adult and elderly patients in use of oncology ostomies. Literature review: Initially, we selected 30 publications that presented the inclusion criteria. Posteriorly, the analysis of the data available in the national and international literature made it possible to describe the current epidemiological perspectives who evidence the growth of new cancer cases associated to changes in the sociodemographic profile of the population. And, concomitantly, with highkight for the sequels caused by antineoplastic treatment, for the ostomization process. This review made it possible to describe the types of ostomies, what possible complications the patient may present and how the process of adaptation to this new condition occurs. Final considerations: The study demonstrates the importance of the multiprofessional approach to quality of life assurance in the biopsychosocial perspective of adult and elderly ostomized oncological patients.
\end{abstract}

Keywords: Ostomy, Neomplsms, Patient care team.

\footnotetext{
${ }^{1}$ Universidade Federal de Santa Maria (UFSM), Rio Grande do Sul - RS.

*E-mail: arielenferigollo2@hotmail.com
} 


\section{RESUMEN}

Objetivo: Realizar una revisión de los aspectos biopsicosociales así como lo rendimento de esquipe multidisciplinario hacia adelante para pacientes adultos y ancianos con câncer y ostomizados. Revisión de literatura: Al principio, se seleccionaron 30 publicaciones que presentó los criterios de inclusión. Posteriormente, el análisis de los datos disponibles en la literatura nacional e internacional ha permitido la descripción epidemiológica de las actuales perspectivas que muestran el crecimiento de nuevos casos de cáncer asociado a los cambios en el perfil demográfico de la población. Así, por ende, el crecimiento de las secuelas causadas por el tratamiento antineoplásico, con énfasis en el proceso de ostomização. Este estudio ha permitido la descripción de los tipos de ostomías, cuáles son las posibles complicaciones que el paciente puede presentar y cómo el proceso de adaptación a esta nueva condición. Consideraciones finales: El estudio demuestra la importancia del enfoque multiprofesional para garantizar la calidad de la vida en la perspectiva biopsicosocial de los pacientes adultos y ancianos con cáncer y ostomizados.

Palabras clave: Estomía, Neoplasias, Equipo de asistencia al paciente.

\section{INTRODUÇÃO}

O câncer é caracterizado com uma doença não transmissível e representa significativo impacto na população mundial, sendo esperado que para o ano de 2030 a incidência deste seja de 21,4 milhões de casos e o número de óbitos de 13,2 milhões (STEWART BW e WILD CP, 2014).

No Brasil, a última estimativa do Instituto Nacional de Câncer José Alencar Gomes da Silva (INCA, 2017), indicou que para 2017 e 2018 a incidência será de 600 mil novos casos por ano. Esta doença tem como característica comum o processo de crescimento e disseminação incontrolado de células. As células cancerosas podem acometer diversas camadas celulares dos tecidos e órgãos, deslocar-se pela corrente sanguínea ou linfática e ainda, espalhar-se para outras regiões do corpo (WHO, 2017).

No Brasil, a maioria dos casos de câncer são diagnosticados em estágios avançados, sendo necessário ser levado em conta as condições de vida da população, as dificuldades de acesso às ações de prevenção e promoção, além do contexto contemporâneo da política de saúde no país. O tratamento das neoplasias pode ser realizado por meio de cirurgia, radioterapia, quimioterapia, imunoterapia, hormonioterapia ou transplante de medula óssea, ainda em muitos casos, é necessário combinar mais de uma modalidade (INCA, 2015).

Sob esse aspecto, dentre as sequelas temporárias e/ou permanentes que o tratamento das neoplasias malignas pode originar estão a confecção de um estoma. Os termos ostomia, ostoma, estoma ou estomia se originam do termo grego stóma, e designam de boca ou abertura (SAMPAIO FAA et al., 2008). Dependendo da parte do corpo onde será realizado o procedimento, as ostomias adquirem um termo específico, sendo classificadas em digestivas (gastrostomia e jejunostomia), intestinais (colostomia, ileostomia), urinárias (urostomia) e respiratórias (traqueostomias). Contudo, apresentam características peculiares no que se refere aos cuidados, complicações e requisitos especiais de adaptação aos estilos de vida (GUTMAN N, 2011).

A presença de uma ostomia pode ocasionar mudanças na vida do paciente, as quais iniciam no momento em que a doença ou condição é descoberta, desencadeando comportamentos de enfrentamento do paciente. A realização deste procedimento pode suscitar inicialmente a concepções negativas referentes ao estoma e seus efeitos sobre aspectos na vida, como alterações no autocuidado, consumo alimentar, hábito intestinal, atividades sociais e sexuais.

É necessário auxílio multiprofissional para que o paciente consiga avançar pelos processos de ajuste à ostomia, até retornar às suas atividades diárias habituais (MUNOZ BM e JIMÉNEZ RP e DIAZ LR, 2010; CHENG F et al., 2013).

A equipe multiprofissional é fundamental, devido à complexidade que envolve o atendimento destes pacientes. Os profissionais podem atuar na perspectiva de viabilizar práticas educativas que envolvem 0 autocuidado e a avaliação das necessidades biopsicossociais do paciente e da família, visando assim a melhoria da qualidade de vida desses pacientes. (CETOLIN SF e BELTRAME V e CETOLIN SK et al., 2013). 
Neste contexto, buscou-se realizar uma revisão narrativa sobre os aspectos biopsicossociais e atuação da esquipe multidisciplinar frente aos pacientes oncológicos ostomizados.

\section{REVISÃO BIBLIOGRÁFICA}

Foram identificados 450 trabalhos, após a leitura dos títulos e dos respectivos resumos com o objetivo de verificar se o conteúdo apresentava proximidade com o tema proposto, restaram 34 estudos. A partir da leitura detalhada destes trabalhos, para melhor compreensão do assunto, os dados foram dispostos em unidades temáticas: câncer e suas implicações na saúde; câncer e ostomias; ostomia: como se adaptar?; a abordagem multidisciplinar na assistência ao paciente oncológico ostomizados.

\section{Câncer e suas implicações na saúde}

A partir da segunda metade da década de 60, na grande parte dos países da América Latina, observouse diminuição da fecundidade e alterações significativas na distribuição etária da população (BRUNELLO A e SANDRI R e EXTERMANN M, 2009). No Brasil, entre 1997 e 2007, notou-se um crescimento de 21,6\% para $47,8 \%$ do grupo com 60 anos ou mais e estima-se que para 2060 estes indivíduos representarão $26,7 \% \%$ da população brasileira (IBGE, 2010).

O envelhecimento populacional é considerado um novo desafio para a saúde pública contemporânea, bem como um fator de risco independente para a oncogênese (FERLAY J e SOERJOMATARAM I e ERVIK M et al., 2013). Idosos representam a maioria dos casos novos e dos óbitos pela doença, a elevada incidência e letalidade observadas atualmente apontam para a necessidade de uma atenção específica a este grupo e suas particularidades. (SANTOS CSVB, 2012).

Ademais, a interação entre diversos fatores endógenos e ambientais pode resultar no desenvolvimento de várias formas de câncer. Estudos epidemiológicos estão, cada vez mais, associando os padrões alimentares observados em diversas regiões do mundo com a prevalência de câncer. Outros fatores ambientais como tabagismo, obesidade, sedentarismo e exposição a tipos específicos de vírus, bactérias e parasitas, além do contato frequente com algumas substâncias carcinogênicas como produtos de carvão e amianto, também estão relacionados com o desenvolvimento de neoplasias (GARÓFOLO A e AVESANI CM e CAMARGO GK et al., 2004).

O crescimento celular desordenado ocasiona a ativação do processo inflamatório e consequente ativação do sistema imunológico pelo organismo, com produção de citocinas e proteínas de fase aguda como proteína$C$ reativa que, resulta no hipermetabolismo, acelerando a perda ponderal (ARGILÉS JM e BUSQUETS FJ e LÓPEZ-SORIANO M et al., 2006).

Assim, as modalidades convencionais para o tratamento antineoplásico incluem cirurgia, quimioterapia, imunoterapia, hormonioterapia, radioterapia e transplante de medula óssea (INCA, 2015). Os tipos de tratamento oncológicos são complexos e envolvem o trabalho de diversos especialistas (IKEMORI EH e OLIVEIRA EH e SERRALHEIRO IF, 2003).

O tratamento quimioterápico se constituiu de medicamentos que controlam ou curam essa patologia, atuando na destruição de células malignas, impedindo a formação de um novo ácido desoxirribonucleico (DNA), bloqueando funções essenciais da célula ou induzindo a apoptose. A dose da quimioterapia é calculada, geralmente, de acordo com o peso e a altura do paciente. Por ser um tratamento sistêmico, todos os tecidos podem ser afetados, embora em graus diferentes (DIAS MV e MORIMOTO IMI, 2006).

A radioterapia é outra modalidade terapêutica capaz de destruir células cancerígenas, empregando feixe de radiações ionizantes. Uma dose previamente estabelecida de radiação é aplicada, por um tempo, no decorrer de determinados dias, a um volume de tecido que engloba o tumor, com o objetivo de matar células tumorais, com o menor dano possível às células saudáveis em volta (ROCHA RC e LEHN C e OLIVEIRA JXA et al., 2008).

A cirurgia é a modalidade na qual o câncer, em sua fase inicial, pode ser controlado e curado cirurgicamente, quando o tratamento cirúrgico é o indicado para o caso. A cirurgia foi o primeiro tratamento 
que alterou significativamente o curso da doença neoplásica e, até hoje, é um dos principais métodos utilizados, sendo ainda muito importante no arsenal terapêutico dos tumores malignos. Pode ser realizada com finalidade diagnóstica, preventiva, curativa ou paliativa (INCA, 2008).

Em relação ao controle e prevenção do câncer foi criado a Portaria № 2.439 criada em 08 de dezembro de 2005, que estabelece a Política Nacional de Atenção Oncológica (PNAO) que prevê ações de promoção, prevenção, diagnóstico, tratamento, reabilitação e cuidados paliativos, a serem implantadas em todas as unidades federadas, respeitadas as competências das três esferas de gestão, definindo instrumentos de ação para intervir na atenção oncológica no país.

Nesse contexto, as equipes multidisciplinares e multiprofissionais inseridas nos serviços de saúde, vinculam essas estratégias em seu cotidiano de trabalho, buscando contribuir para a construção de hábitos saudáveis, práticas educativas e melhorias na qualidade de vida dos pacientes (BRASIL, 2005).

Nesta perspectiva, é que os benefícios decorrentes das modificações no estilo de vida, incluindo-se as modificações dietéticas, para redução mundial dos coeficientes de incidência e mortalidade de câncer, devem ser instigadas. Além disso, a adoção de hábitos saudáveis, incluindo a alimentação, constitui fator de proteção contra o desenvolvimento de vários cânceres.

Entretanto, os nutrientes específicos, responsáveis pelos mecanismos anticarcinogênicos, ainda não foram completamente identificados, fazendo-se necessária a pesquisa, principalmente, no âmbito nacional (GARÓFOLO A e AVESANI CM e CAMARGO GK et al., 2004).

\section{$O$ câncer e as ostomias}

O paciente ostomizado ou estomizado se caracteriza por ser aquele que necessitou passar por uma intervenção cirúrgica para realizar no corpo uma abertura alternativa, que servirá de comunicação com o meio exterior, para a saída de fezes ou urina, assim como para auxiliar na respiração ou na alimentação deste paciente (ONCOGUIA, 2016).

Para essa abertura, designa-se o termo estoma ou ostomia (ONCOGUIA, 2016). Podem ser temporárias, se estabelecem um determinado período de tempo ou definitivas quando o paciente terá que conviver por tempo indeterminado (GEMELLI LMG e ZAGO MMF, 2002; DI GESARO A, 2012).

Em 350 aC surgiram as primeiras informações sobre as ostomias. Relatadas na Bíblia, elas citam uma passagem onde Praxógoras de Kos teria realizado esta cirurgia, em um ferimento abdominal (CASCAIS AFMV; MARTINI JG; ALMEIDA PJS, 2007). Já em 1709, um cirurgião alemão, Lorenz Heister, teria realizado uma operação de enterostomia em soldados com ferimentos intestinais (CASCAIS AFMV; MARTINI JG; ALMEIDA PJS, 2007). Porém, é no início da década de 1950, conhecida como a "era moderna das ostomias", que ocorreu o aprimoramento desta técnica cirúrgica (CASCAIS AFMV; MARTINI JG; ALMEIDA PJS, 2007).

Assim, quanto ao tipo, as ostomias podem ser classificadas em: Colostomia e ileostomia, onde ambas são intervenções cirúrgicas, uma realizada no intestino grosso e a outra no intestino delgado. Ambas possuem o intuito de criar uma abertura artificial para a saída do conteúdo fecal, através da exteriorização de uma fração intestinal (ONCOGUIA, 2016); A urostomia, no entanto, consiste na criação de um trajeto de drenagem da urina pela parede abdominal, com o objetivo de preservar a função renal (ONCOGUIA, 2016); Já a gastrostomia consiste na realização de uma comunicação do estômago com o meio exterior (ONCOGUIA, 2016). Diferentemente da Jejunostomia, que estabelece um acesso alternativo para o o jejuno proximal. Por fim, a traqueostomia caracteriza-se por ser um procedimento cirúrgico realizado para criar uma comunicação da traqueia com o ambiente externo, tendo por objetivo a melhora do fluxo respiratório (BRASIL, 2009).

Relacionando a ostomia e o câncer, deve-se considerar a agressividade que o tratamento oncológico ocasiona ao corpo, pois, os efeitos terapêuticos e tóxicos dos tratamentos antineoplásicos dependem do tempo de exposição, da concentração plasmática da droga, da dose de radiação ionizante, volume e área que será irradiada, além da aplicação concomitante ou não da quimioterapia e radioterapia.

Tais modalidades terapêuticas podem ocasionar diversos eventos adversos, principalmente aqueles ligados diretamente ao trato gastrointestinal, culminando com uma possível redução da ingestão alimentar e

REAS/EJCH | Vol.12(8) | e3354 | DOI: https://doi.org/10.25248/reas.e3354.2020 Página 4 de 8 
consequentemente depleção do estado nutricional, sendo necessário o uso de vias alternativas de alimentação, como gastrostomia ou jejunostomia (HILL A e KISS N e HODGSON B et al., 2011; LIS CG e GUPTA D e GRUTSCH JF, 2009).

No que diz respeito aos pacientes ostomizadas, as práticas alimentares podem provocar repercussões bastante significativas, podendo influenciar de forma positiva ou negativa no processo de adaptação à sua nova condição de vida.

Desta forma, oferecer cuidado a esses pacientes requer a percepção das singularidades do indivíduo, compreendendo as fases de enfrentamento e adaptação tanto em relação aos aspectos emocionais, quanto às mudanças físicas decorrentes desta nova condição (DA SILVA AL e SHIMIZU HE, 2007).

O paciente ostomizado poderá ou não manifestar repulsa e relutância em adaptar-se com esta condição, contudo, cada indivíduo é único, sendo assim este processo poderá apresentar variância. A condição de saúde da pessoa que vive com uma ostomia caracteriza-se como uma situação crônica ao longo da sua vida, tornando necessário que esta desenvolva um conjunto de aprendizagens e estratégias que lhe permitam ampliar competências de autocuidado, convivência social, além dos aspectos psicológicos, para que possa gerir a situação do processo saúde-doença. Neste sentido, a família é fundamental no decorrer desta trajetória, na mesma medida que o apoio das equipes de saúde envolvidas neste contexto (SANTOS CSVB, 2012).

A família apresenta-se como suporte concreto, e que contribui para dar-lhes sentido à luta pela vida. A família assume o cuidado físico e emocional, além de oferecer proteção, conforto e afeto. Neste sentido, é que os apoios funcionam para minimizar o sofrimento, e nesta perspectiva, os profissionais de saúde devem estimular os pacientes a manifestarem sua sensibilidade para que juntos encontrem estratégias que facilitem a compreensão e o enfrentamento do momento vivido, pelas pessoas estomizadas a fim de trabalhar a melhoria da sua qualidade de vida (DA SILVA AL e SHIMIZU HE, 2007).

\section{Ostomia: como se adaptar?}

O aprendizado dentro dessa nova relizada configure-se como um desafio, exigindo, sobretudo, respeito às mágoas, ressentimentos, revoltas e perdas, para o avanço que visa a aceitação da nova condição de vida que se apresenta após a ostomia (CETOLIN SF e BELTRAME V e CETOLIN SK et al., 2013). Conviver com a ostomia pode causar sentimentos negativos como 0 medo, além de possíveis desconfortos e constrangimentos. CETOLIN SF e BELTRAME V e CETOLIN SK et al., 2013).

Nesse sentido, o apoio da família e da sociedade à pessoa portadora de uma ostomia é importante para auxiliá-la na recuperação de sua autoestima e à volta da sua convivência social, objetivando assim, alcançar uma melhor qualidade de vida. (CETOLIN SF e BELTRAME V e CETOLIN SK et al., 2013).

A adaptação do paciente ao uso de algum tipo de ostomia requer cuidados específicos, para que essa possa the oferecer conforto e segurança. No entanto, este procedimento pode desencadear sentimentos conflituosos e preocupantes, que abarcam aspectos fisiológicos, psicológicos e sociais (INCA, 2003).

Sob esse enfoque, conforme o Departamento de Psiquiatria UNIFESP/EPM o paciente que necessita submeter-se a ostomia, sofre várias modificações no seu cotidiano, abarcando aspectos biopsicossociais do sujeito, fato que está estreitamente relacionado ao sofrimento, a dor, a degeneração, incertezas quanto ao futuro, mitos relacionados a ele, medo da rejeição, entre outros (ALVES LC, 2000).

O estado emocional do paciente pós-cirúrgico, pode acarretar em ansiedade, agressividade, depressão, receio de ficar impossibilitado de trabalhar, bem como receoso em relação às atividades sociais e sexuais. Isso acontece em decorrência da dificuldade em conviver com uma aparência diferente, causada pela colocação da ostomia.

O impacto da presença da ostomia traz consigo, o impacto no modo como o sujeito percebe o próprio corpo e está relacionado a como uma pessoa valoriza suas habilidades físicas, interpessoais, papéis desempenhados dentro da família e imagem corporal, tal fato está atrelado a aspectos culturais de como o indivíduo constrói sua identidade ao longo da vida, uma imagem de seu próprio, a qual está vinculada a beleza 
e vigor, e onde aqueles que não fazem parte deste grupo podem experimentar o significativo sentimento de rejeição (CREMA E e SILVA JA, 1997).

Para a United Ostomy Association (2004), a adaptação à condição de portador de ostomias é um processo que requer paciência, persistência e conhecimento sobre os cuidados que envolvem sua colocação, e está vinculado à condição sistêmica, hábitos de vida, e a personalidade de cada paciente.

Neste contexto, o paciente submetido a colocação de uma ostomia, procedimento doloroso e agressivo, o qual altera tanto sua fisiologia, quanto sua imagem corporal, sexualidade, além de outras tantas modificações em sua vida, tem se constituído como um desafio para os cuidadores da equipe multidisciplinar que 0 atendem.

\section{Abordagem multidisciplinar na assistência ao paciente oncológico ostomizado}

Ao reconhecer o câncer como um problema de saúde pública em decorrência dos altos índices de novos casos e considerando que esta doença traz consigo implicações de natureza física, emocional, social e econômica para a vida dos sujeitos, esta condição exige $o$ atendimento de equipes multidisciplinares e multiprofissionais capacitadas.

No Brasil, tem se identificado que a maioria dos casos de câncer são diagnosticados em estágios avançados, sendo necessário ser levado em conta as condições de vida da população, as dificuldades de acesso às ações de prevenção e promoção, além do contexto contemporâneo da política de saúde no país (INCA, 2012).

Quando se trata da intervenção das equipes de saúde em pacientes oncológicos ostomizados, é preciso evidenciar que a importância de profissionais capacitados, envolvidos nas orientações dos cuidados aos pacientes e no enfrentamento e a adaptação à sua nova condição. Assim, o acompanhamento aos pacientes ostomizados e seus cuidadores é essencial, pois os mesmos irão necessitar de apoio para enfrentar as transformações resultantes do uso da ostomia (CETOLIN SF e BELTRAME V e CETOLIN SK et al., 2013).

Com relação aos Serviços de Atenção à Saúde das Pessoas Ostomizadas no SUS, cabe destacar, a Portaria no 400 de 16 de novembro de 2009, que considera a necessidade de garantir aos ostomizados a atenção integral à saúde por meio de intervenções especializadas de natureza interdisciplinar e que o pleno atendimento às suas necessidades depende da qualificação dos processos de atenção que incluem prescrição, fornecimento e adequação de equipamentos coletores e adjuvantes de proteção e segurança (BRASIL, 2009).

Assim, a equipe multiprofissional que atende o paciente oncológico ostomizado deverá estar atenta às demandas que este processo poderá desencadear. É importante também, que se estabeleçam vínculos, possibilitando assim a abordagem das necessidades decorrentes das mudanças nos aspectos sociais, familiares, nutricionais e psicológicos desses pacientes. Desse modo, a equipe multiprofissional poderá contribuir de forma positiva na qualidade de vida do paciente oncológico ostomizado (CETOLIN SF e BELTRAME V e CETOLIN SK et al., 2013).

Nesse sentido, observa-se a importância do papel que exerce a equipe multiprofissional, ao melhorar a qualidade de vida do paciente e do seu núcleo familiar. Também destaca-se que, a forma de enfrentamento e adaptação à uma nova realidade é individual e singular de cada pessoa, podendo vir acompanhada de sofrimento, dor, incertezas, mitos e medos. (CETOLIN SF e BELTRAME V e CETOLIN SK et al., 2013). Assim, o acompanhamento aos ostomizados torna-se indispensável, havendo a necessidade de apoio para 0 enfrentamento das transformações resultantes da ostomia (CETOLIN SF e BELTRAME V e CETOLIN SK et al., 2013).

Complementa-se ainda, que a utilização de estratégias que atendam as expectativas do momento vivenciado por pacientes com câncer e ostomia, precisam ser desenvolvidas para alcançar suas finalidades. Reforça-se, que é importante ter motivação à desenvolver atividades que possam contribuir com a capacitação da equipe multiprofissional, tendo em mente que o conhecimento científico é a base para o fazer e as deficiências a serem superadas devem considerar os níveis: cognitivo, afetivo e psicomotor relacionados a estomaterapia (GEMELLI LMG e ZAGO MMF, 2002). 


\section{CONSIDERAÇÕES FINAIS}

As implicações físicas, sociais, psicológicas e familiares demandam atenção especializada em saúde. Ao reconhecer os processos de adaptação e a reabilitação aos ostomizados articulados em equipe multiprofissional, compreende-se que estes contribuem para restituir as atividades da rotina, impactando significamente na reinserção social desses sujeitos, o que melhora sua qualidade de vida diante do impacto da realização de uma ostomia. Conclui-se, então, que as repercussões impostas por ostomia na vida dos pacientes em tratamento oncológico, demandam uma abordagem multiprofissional, pois cada profissional atua integrando os conhecimentos de sua área específica aos demais, buscando a construção de um plano terapêutico qualificado e especializado, para o enfrentamento a essa nova condição.

\section{REFERÊNCIAS}

1. ALVES LC. Correlação entre adaptação psicossocial à colostomia permanente e resposta psicológica ao câncer. Órgão Oficial do Centro de Estudos - Departamento de Psiquiatria-UNIFESP/EPM, 2000; 33(4).

2. ARGILÉS JM, et al. Fisiopatología de la caquexia neoplásica. Nutrição Hospitalar, 2006; 21(3): 4-9.

3. BRASIL. Ministério da Saúde. Instituto nacional de Câncer. Portaria no 2.439 de 8 de dezembro de 2005.

4. BRASIL. Ministério da Saúde. Secretaria de Atenção à Saúde Portaria n 400 de 16 de novembro de 2009.

5. BRASIL. Ministério da Saúde. Estimativa 2016: incidência de câncer no Brasil. 2015; Rio de Janeiro: INCA.

6. BRASIL. Ministério da Saúde. Estimativa 2008: Incidência de câncer no Brasil. 2007; Rio de Janeiro: INCA.

7. BRASIL. Ministério da Saúde. Orientação sobre Ostomias. 2003; Brasília - DF: INCA.

8. BRUNELLO A, SANDRI R, EXTERMANN M. Multidimensional geriatric evaluation for older cancer patients as a clinical and research tool. Cancer Treatment Reviews, 2009; 35: 487- 492.

9. CASCAIS AFMV, MARTINI JG, ALMEIDA PJS. O impacto da ostomia no processo de viver humano. Revista Texto Contexto Enfermagem, 2007; 16(1): 163-167.

10. CETOLIN SF, et al. Dinâmica sócio-familiar com pacientes portadores de ostomia intestinal definitiva. ABCD. Arquivos Brasileiros de Cirurgia Digestiva (São Paulo), 2013; 26(3): 170-172.

11. CHENG F, et al. The correlation between ostomy knowledge and self-care ability with psychosocial adjustment in Chinese patients with a permanent colostomy: a descriptive study. Ostomy Wound Manage, 2013; 59(7): 35-8.

12. CREMA E, MARTINS JA. Complicações dos estomas intestinais. In: CREMA, E.; SILVA, R. Estornas: uma abordagem interdisciplinar. Uberaba, Editora Pinti, 1997; 7: 89-106.

13. DA SILVA AL, SHIMIZU HE. O significado da mudança no modo de vida da pessoa com estomia intestinal definitiva. Revista Latino-americana de enfermagem, 2007; 14(4).

14. DIAS MV, MORIMOTO IMI. O grau de interferência dos sintomas gastrintestinais no estado nutricional do paciente com câncer em tratamento quimioterápico. Revista Brasileira Nutrição Clínica, 2006; 21(3): 211-218.

15. DI GESARO A. Self-care and patient empowerment in stoma management. Gastrointestinal Nursing, 2012; 10(2): 1923.

16. FERLAY J, et al. Cancer Incidence and Mortality Worldwide: IARC Cancer Base, 2013; 11.

17. GARÓFOLO A, et al. Dieta e câncer: um enfoque epidemiológico. Revista Nutrição, Campinas, SP, 2004 ; 17: 491505.

18. GEMELLI LMG, ZAGO MMF. A interpretação do cuidado com o ostomizado na visão do enfermeiro: um estudo de caso. Revista Latino-americana de enfermagem, 2002; 10(1): 34-40.

19. GUTMAN N. Guia de colostomia. Northfield: United Ostomy Associations of América. 2011.

20. HILL A, et al. Associations between nutritional status, weight loss, radiotherapy treatment toxicity and treatment outcomes in gastrointestinal cancer patients. Clinical nutrition, 2011; 30(1): 92-98.

21. INSTITUTO BRASILEIRO DE GEOGRAFIA E ESTATíSTICA (IBGE). Atlas do censo demográfico 2010. Rio de Janeiro, 2013.

22. IKEMORI EH, OLIVEIRA EHI, SERRALHEIRO IF. Nutrição em oncologia. São Paulo: Lemar, $2003 ; 471$.

23. INSTITUTO NACIONAL DE CÂNCER JOSÉ ALENCAR GOMES DA SILVA (INCA). ABC do câncer: abordagens básicas para o controle do câncer. Rio de Janeiro: Inca. 2012; 2. ed. rev.

24. INSTITUTO NACIONAL DE CÂNCER JOSÉ ALENCAR GOMES DA SILVA (INCA). Estimativa 2018: incidência de câncer no Brasil. 2017; Rio de Janeiro: INCA.

25. INTERNATIONAL AGENCY FOR RESEARCH ON CANCER - IARC. Pharmaceuticals: a review of human carcinogens, 2012.

26. LIS CG, GUPTA D, GRUTSCH JF. Can anorexia predict patient satisfaction with quality of life in advanced cancer? Supportive Care in Cancer, 2009; 17(2): 129-135.

27. MUNOZ BM, et al. El proceso de afrontamiento en personas recientemente ostomizadas. Index Enferm, Granada, 2010; 19(2-3): 115-119.

28. ONCOGUIA. Instituto Oncoguia. In: Ostomizados. 2016. http://www.oncoguia.org.br/conteudo/ostomizados/1853/15/. Acesso em: 14. abr. 2020.

29. ROCHA RC, et al. Incidência de osteorradionecrose em pacientes com câncer de boca tratados com radioterapia exclusiva ou associação com cirurgia. Revista Brasileira de Cirurgia de Cabeça e Pescoço, 2008; 37(2): 91-94. 
30. SAMPAIO FAA, et al. Assistência de enfermagem a paciente com colostomia: aplicação da teoria de Orem. Acta Paulista de Enfermagem, 2008; 21(1): 94-100.

31. SANTOS CSVB. Nota introdutória. In Associação Portuguesa de Enfermeiros de Cuidados em Estomaterapia, Estomaterapia o saber e o cuidar. Lisboa: Lidel. 2012.

32. STEWART BW, WILD CP. (Ed.). World Cancer Report: 2014. Lyon: IARC, 2014.

33. UNITED OSTOMY ASSOCIATIONS OF AMERICA (UOAA). In: Colostomy guide [online]. Kennebunk (ME): United Ostomy Associations of America. $2011 . \quad$ Disponível em: http://www.ostomy.org/uploaded/files/ostomy_info/ColostomyGuide.pdf?direct=1. Acesso em: 14. abr. 2020.

34. WORLD HEALTH ORGANIZATION. Tracking universal health coverage: 2017 Global Monitoring Report. Geneva: World Bank/World Health Organization; 2017. 